\title{
Los estudios demográficos a inicios del siglo XXI
}

En las últimas décadas las investigaciones sociodemográficas y, en general, los estudios de población han ampliado, diversificado y enriquecido sus enfoques a partir de la incorporación de la perspectiva de género, la inclusión de las dimensiones étnico-raciales, las consideraciones que marcan las diferencias etarias y el reconocimiento de la diversidad sexual, entre otros. Esta apertura del campo de la investigación sociodemográfica ha favorecido un mayor reconocimiento de las desigualdades sociales y de la alteridad, y ha permitido instalar los estudios sociodemográficos en un terreno explicativo de primer orden.

La ampliación del campo sociodemográfico se ha expresado en un mayor fortalecimiento en la identificación de patrones y tendencias sociales y ha contribuido de manera significativa a la comprensión del cambio social en las sociedades contemporáneas. En virtud de la interesante dinámica que se expresa en este campo del conocimiento, la revista sociedad y economía presenta la edición de su número 29, con el tema central: Los estudios sociodemográficos a inicios del siglo XXI. Los ocho artículos seleccionados entre los que respondieron a la convocatoria constituyen una significativa muestra de la diversidad y complejidad que implica la incorporación de los emergentes enfoques analíticos, tanto en aquellos artículos que enfatizan en uno de estos enfoques como en aquellos que, de manera innovadora, avanzan en sus articulaciones y muestran sus intersecciones.

En el artículo “Jóvenes de hoy, adolescentes de ayer en el Uruguay: maternidad y desempeños", de Carmen Varela Petito y Cecilia Lara, se discuten las implicaciones del embarazo adolescente a partir de un riguroso análisis de los desempeños sociales de dos generaciones de mujeres uruguayas, una vez que están próximas a finalizar la juventud y a ingresar a la adultez; diferenciando entre aquellas que fueron madres en la adolescencia y aquellas que no lo fueron. El sugerente diseño de investigación y el aprovechamiento de la información recabada en la Encuesta Nacional de Juventud en Uruguay de 1990 y 2008 permite constatar el estancamiento en el descenso de la fecundidad adolescente en Uruguay y plantea la pregunta en torno a si la maternidad en la adolescencia es un fenómeno instaurado y difícil de cambiar. De igual forma, el análisis que se presenta en este artículo con respecto a los desempeños y logros al final de la juventud de las mujeres que tuvieron su primer hijo en la adolescencia permite identificar el peso diferencial de un conjunto de desventajas, en el nivel educativo alcanzado y en la inserción laboral, asociadas a la maternidad en la adolescencia con respecto a las mujeres que pospusieron la maternidad para etapas posteriores del ciclo de vida.

El debate contemporáneo en torno al reconocimiento y la aceptación de la paternidad gay ha favorecido tanto la emergencia de un sinnúmero de nuevos escenarios para la investigación social como la apertura de los estudios sociodemográficos a la visibilización del otro, el sujeto con prácticas homoeróticas, el homosexual clásico o el gay contemporáneo. Sebastián Giraldo Aguirre, en su 
artículo "Prácticas de paternidad de algunos varones gay de la Ciudad de México. Entre tabúes y nuevas apuestas para su ejercicio", nos presenta un conjunto de reflexiones en torno al ejercicio de la paternidad fundamentada en la experiencia vivida y documentada a partir de entrevistas realizadas por el autor a un selecto grupo de cuatro padres que se reconocen como hombres con orientación sexual gay, cohabitan con su hijos/as, tienen alta escolaridad, pertenecen a la clase media y se definen como activistas por la diversidad sexual. La apuesta analítica del autor diferencia entre aquellas dimensiones asociadas, en mayor medida, con el rol paterno -el tiempo dedicado al cuidado de sus hijos/as, la proveeduría económica y la autoridad- de otras dimensiones que, de manera innovadora, introduce el autor para presentar un análisis exploratorio acerca de la asociación entre la orientación sexual y los valores inculcados a los hijos/as y la socialización de género.

De otro lado, en las últimas décadas, los estudios sobre el uso del tiempo de hombres y mujeres en relación con el trabajo remunerado, el trabajo no remunerado y las actividades de ocio han enfatizado en la importancia de considerar las diferencias por edad, condición conyugal, nivel de escolaridad y posición ocupacional, entre otras y han señalado la persistencia en las inequidades de género en la distribución de las actividades domésticas y de cuidado. El artículo "Participación y tiempo en actividades cotidianas de hombres y mujeres vinculados al mercado laboral en México", de María Viridiana Sosa Márquez y Rosa Patricia Román Reyes, examina en detalle la información diferenciada por sexo, capturada en la Encuesta Nacional sobre Uso del Tiempo en México, en 2009. Fundamentadas en el análisis de esta información, las autoras discuten la organización social del uso del tiempo de hombres y mujeres que trabajan de manera remunerada y analizan su relación con los patrones familiares tradicionales y emergentes en el México de inicios del siglo XXI.

El análisis comparativo de las características y patrones que adquiere el trabajo infantil rural asalariado en dos complejos agroindustriales (CAI) altamente modernizados en sus procesos de producción en correspondencia con las dinámicas demandantes de la competitividad productiva en el marco de la economía global: la actividad tabacalera en la provincia argentina de Jujuy y la floricultura comercial de exportación en la región sur del Estado de México, da lugar al artículo de Sarai Miranda Juárez y Daniel Alberto Re. Este análisis comparativo en contextos rurales de los procesos de inserción laboral temprana de este grupo poblacional identifica que el modelo flexible de gestión de la fuerza de trabajo establece la "ayuda" familiar y el trabajo infantil como uno de sus principales rasgos para enfrentar la estacionalidad y segmentación propia de los procesos productivos estudiados.

La intensificación de la migración altamente calificada y el predominio de estos flujos Sur-Norte constituyen una de las características de la movilidad global contemporánea. El análisis de los principales patrones demográficos de la población colombiana altamente calificada residente en Estados Unidos y la identificación de las principales tendencias que presenta este flujo migratorio entre 2000 y 2011 es abordado en el artículo "La población inmigrante calificada colombiana residente en Estados Unidos”, presentado por Rosa Emilia Bermúdez Rico, a partir del procesamiento de la información disponible en The American 
Community Survey. El análisis del acumulado de inmigrantes calificados colombianos en ese país le permite a la autora dimensionar el dinamismo alcanzado por este grupo poblacional en Estados Unidos, así como subrayar su mayor feminización y establecer pautas de aproximación y diferenciación de la población calificada colombiana con respecto a otras poblaciones tanto de nativos como de inmigrantes en Estados Unidos.

De otro lado, durante las últimas décadas las mujeres de los países latinoamericanos intensificación de manera sostenida los flujos de migración hacia España. En su mayoría estas mujeres andinas se movilizan para insertarse en el trabajo doméstico y de cuidado en ese país. El artículo "Los migrantes colombianos en la prensa colombiana: Una lectura de género 1990-2006", escrito por Victoria Elena Bazurto Botero, registra y analiza las representaciones creadas en torno a las mujeres migrantes a través del discurso de los periódicos El Tiempo y El Espectador. Este análisis subraya en el escaso reconocimiento que desde estas fuentes se otorga a los proyectos migratorios de las mujeres colombianas, en contraste con la persistencia en las representaciones culturales negativas que enfatizan en las implicaciones que tiene la migración de la mujer en las familias y en los hijos y las hijas.

La inclusión de la condición étnico-racial en el análisis sociodemográfico ha representado una importante contribución para la estimación y el reconocimiento de las desigualdades sociales persistentes en las sociedades contemporáneas. Carlos Viáfara López y Nini Johanna Serna Alvarado presentan en su artículo "Desigualdad de oportunidades educativas en la población de 20 a 29 años de Brasil y Colombia, según autoclasificación étnico-racial" estimaciones de la menor probabilidad que tienen los afrodescendientes tanto en Colombia como en Brasil de alcanzar un nivel educativo más alto que la población no afrodescendiente.

Para finalizar la edición de este número de la Revista sociedad y economía, se ha incluido el artículo "Política de Discapacidad e Inclusión de la Universidad del Valle: un proceso participativo” de autoría de Mónica María Carvajal Osorio, en el que se presenta la sistematización de la construcción de una política pública para la atención a un grupo poblacional en franca minoría y desventaja en el acceso y la permanencia en la educación superior. Para el desarrollo del ejercicio de sistematización se acudió a la revisión y análisis de fuentes documentales.

Esperamos que este diverso y plural panorama de los estudios sociodemográficos y de población, de múltiples nacionalidades y temáticas novedosas con enfoques emergentes seleccionados para la edición de este número de la Revista sociedad y economía, brinde mayores elementos para el reconocimiento de patrones y tendencias emergentes en las sociedades de hoy.

Comité editorial Revista sociedad y economía 